\title{
An Implantaten verankerter, herausnehmbarer Zahnersatz
}

\begin{abstract}
Leider ist es auch heute kaum einem Patienten gegönnt, seine eigenen Zähne bis ins hohe Alter zu behalten, so dass mit einem herausnehmbaren Zahnersatz flexibel und einfach die Kaufunktion wiederhergestellt werden kann. Die Versorgung des Unterkiefers stellt dem Zahnarzt jedoch die Aufgabe, der Prothese einen festen Halt zu geben. Dentale Implantate haben sich zur Lösung dieses Problems als zweckmäßig erwiesen.
\end{abstract}

Eine Vielzahl unterschiedlicher Retentionssysteme werden aktuell zur Verankerung der Implantat-getragenen prothetischen Suprakonstruktion verwendet. Sie verleihen der Prothetik eine gute Unterstützung und Stabilität. Trotz einer kleinen Anzahl an Implantaten kann so die Kaufunktion verbessert werden. $\mathrm{Zu}$ dem verhindern die Implantate die Resorption des Alveolarkamms, und häufig kann sogar die bereits existierende Prothetik nach Integration einer Matritze wiederverwendet werden. Grundsätzlich können die Retentionstypen in Einzelretentionen verblockte Retentionen eingeteilt werden. Ein typisches Beispiel sind Dolder-Stegretentionen, die die Implantate durch die Rotationsmöglichkeit der Suprakonstruktion und die Abstützung auf der Mukosa entlasten. Beispiele für Einzelretentionen sind Kugelknopfverankerungen, ERA, Magnete oder Locatoren. Sie sind mit jedem einzelnen Implantat mechanisch über ein $\mathrm{Pa}$ tritzen-/Matritzensystem verbunden.

\section{Krafteinwirkung sollte minimiert werden}

Welche prothetische Retention ist geeignet, die Implantate bestmöglich vor einer Überbelastung zu schützen? Einige Studien, zuletzt eine In-vitro-Studie von Yoo et al. 2017, konnten eine geringere Belastung der einzelnen Implantate bei Einzelretentionen nachweisen. Dies wird mit der Fähigkeit der Locator-

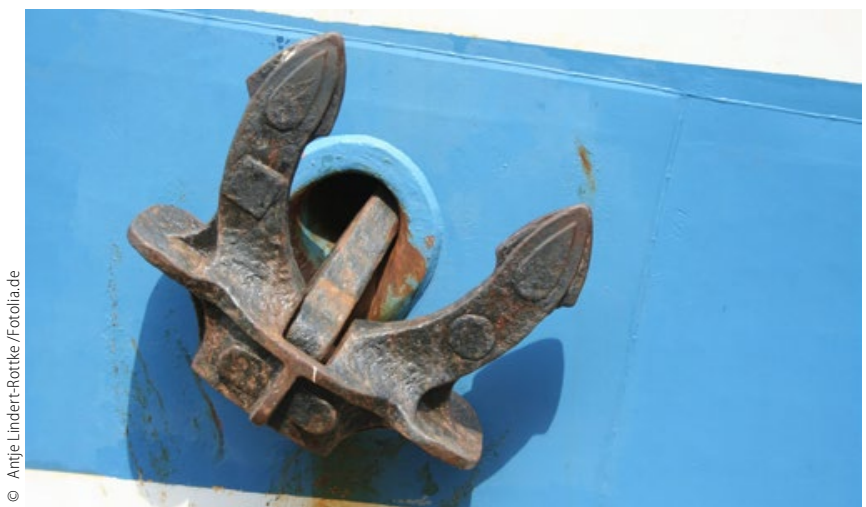

retentionen erklärt, sich zu deformieren und auf diese Weise die vertikalen Kaukräfte zu absorbieren. Zudem ermöglicht die Einzelverankerung die physiologische Verformung des Unterkiefers während der Kaufunktion. Eine Stegverbindung fixiert hingegen die Implantate primär zueinander, so dass bei einer Verformung des Unterkiefers horizontale Kräfte auf die Implantate wirken. Auch eine maximale Extension der Prothesenbasis minimiert die Kräfte an den Implantaten, da die Schleimhaut vertikale Kaukräfte ebenfalls aufnehmen kann. Am meisten profitiert das endständige Implantat in der Zahnreihe von einer dorsalen Prothesenextension.

Da die Überbelastung von Implantaten eine häufige Ursache von Verlusten darstellt, sollte die Krafteinwirkung minimiert werden. Zusammenfassend leistet die Extension der Prothesenbasis und die Verwendung von Einzelversorgungen zur Retention der Suprakonstruktion auf den Implantaten einen wichtigen Beitrag.

\section{Erratum}

In der vergangenen DFZ-Ausgabe ist in dem Artikel „Fortschritte bei der Behandlung initialer Karies - Resininfiltration, Fluoridierung, Nano-Hydroxylapatitbehandlung" ein Schreibfehler passiert. Es stand dort fälschlicherweise "Nano-Hydrylapatitbehandlung".

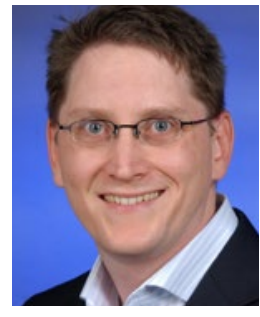

Prof. Dr. Dr. Felix P. Koch

Autor des Wissenschaftlichen Infodienstes, Lehrbeauftragter der Uniklinik Frankfurt, Praxisklinik für Mund-, Kiefer- und plastische Gesichtschirurgie in Wiesbaden Mail:wid@fvdz.de 